\title{
Gefitinib-induced interstitial pneumonia: A case report and review of the literature
}

\author{
CHANGQIN LUO ${ }^{1}$, MEILING LV ${ }^{1}$, YUYAO LI ${ }^{1}$, PEIJUN LIU $^{2}$ and JIN YANG ${ }^{1}$ \\ ${ }^{1}$ Department of Clinical Oncology and ${ }^{2}$ Center for Translational Medicine, The First Affiliated Hospital \\ of Xi'an Jiaotong University College of Medicine, Xi'an, Shaanxi 710061, P.R. China
}

Received May 26, 2013; Accepted September 25, 2013

DOI: $10.3892 /$ etm.2014.1495

\begin{abstract}
The aim of this study was to explore the clinical characteristics of and treatment strategies for interstitial pneumonia induced by gefitinib in patients with advanced non-small cell lung cancer (NSCLC). The detailed clinical data of one patient with NSCLC and gefitinib-induced interstitial pneumonia were compiled and a review of relevant previous studies was performed. Based on this case report and the review, the clinical characteristics, mechanisms and treatment strategies of this rare disease were analyzed. The analyses showed that older, male patients with a long smoking history, high smoking index and adenocarcinoma (particularly bronchoalveolar carcinoma) were more likely to suffer from interstitial pneumonia while taking gefitinib. The onset time of interstitial pneumonia was 1-2 months subsequent to gefitinib administration. The clinical manifestations included chest tightness, shortness of breath, progressive dyspnea, severe hypoxemia and respiratory failure. Diffuse infiltration and alveolar interstitial shadows were observed on the chest tomography scan. In such circumstances, a timely judgment is required, in addition to the withdrawal of gefitinib treatment and the administration of high-dose glucocorticoids, as well as oxygen inhalation and anti-infective therapies, in order to relieve the symptoms. In conclusion, following the onset of gefitinib-induced interstitial pneumonia, the discontinuation of gefitinib is likely to alleviate the suffering of the majority of patients. Early interstitial pneumonia is not an absolute index for the permanent discontinuation of gefitinib treatment. It is necessary to comprehensively consider the benefits and hazards of gefitinib for the patients.
\end{abstract}

Correspondence to: Dr Jin Yang, Department of Clinical Oncology, The First Affiliated Hospital of Xi'an Jiaotong University College of Medicine, No. 277 Yanta District, Xi'an, Shaanxi 710061, P.R. China

E-mail: cqdoccn@yeah.net

Key words: gefitinib, interstitial pneumonia, glucocorticoid

\section{Introduction}

Gefitinib, an epidermal growth factor receptor (EGFR) type 1 tyrosine kinase inhibitor, blocks the signal transduction pathway implicated in the proliferation and survival of cancer cells (1). The use of gefitinib to treat patients with advanced non-small-cell-lung cancer (NSCLC) has raised significant concern among physicians. Gefitinib is better-tolerated and less toxic than conventional cytotoxic drugs; however, gefitinib-induced interstitial lung disease (ILD) has been demonstrated to be a serious adverse effect (2). Phase II studies showed that the objective response rates achieved after gefitinib treatment were between 10 and $20 \%$, with minimal toxicities; mainly an acne-like rash and mild diarrhea, in patients with recurrent non-small cell lung cancer (the IDEAL trials) (3). In subsequent phase III trials, the addition of gefitinib to standard platinum-based chemotherapy failed to demonstrate a survival advantage in patients with untreated non-small cell lung cancer (the INTACT trials) $(4,5)$. The worldwide incidence of ILD was approximately $1 \%$ (2\% in the Japanese post-marketing experience and approximately $0.3 \%$ in a United States expanded access program). The median time to onset of ILD was 24 days in the Japan group and 42 days in the United States group. Approximately one-third of all ILD cases caused by gefitinib have been fatal (6). In this study, a case of gefitinib-induced interstitial pneumonia is described and previous case reports between 2003 and 2011 are reviewed, with the aims of summarizing the clinical features, mechanisms and treatment strategies of gefitinib-induced interstitial pneumonia and providing a reference for medication safety in the clinical treatment of NSCLC.

\section{Case report}

A 62-year-old man, complaining of fever and shortness of breath $>1$ year subsequent to surgery for right-sided NSCLC, was admitted to the First Affiliated Hospital of Xi'an Jiaotong University (Xi'an, China). In July 2010, the patient was revealed to have a lesion in the right upper lobe of the lung in a physical examination. In August 2010, upper right lobe resection and mediastinal lymph node dissections were performed. The tumor measured $3.5 \times 3.0 \times 2.0 \mathrm{~cm}$. The pathological analysis of the resected upper right lung showed that the patient was suffering from differentiated adenocarcinoma and bronchioloalveolar carcinoma. The lung membrane, bronchial stump 
A

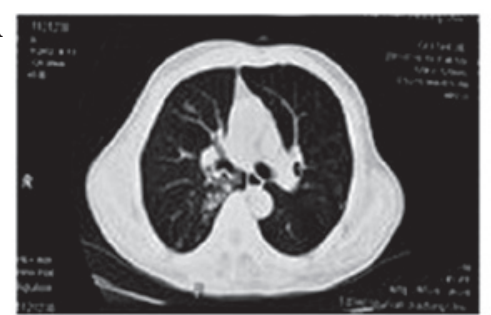

D

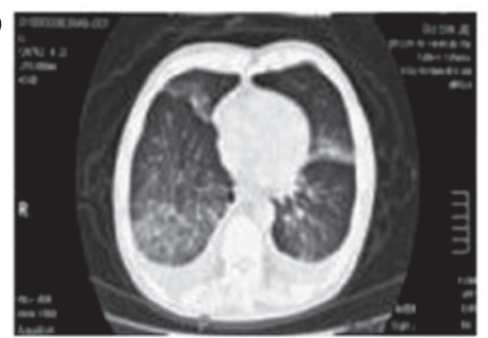

G

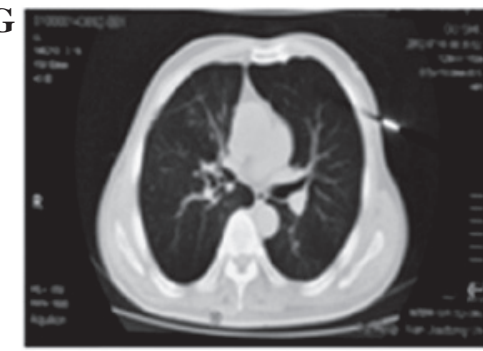

B

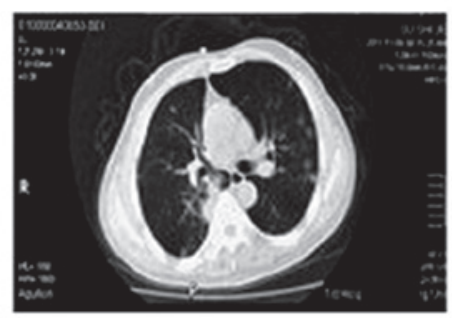

$\mathbf{E}$

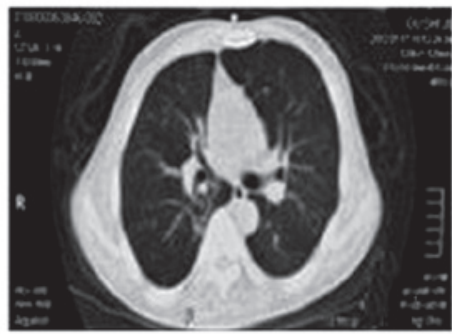

H

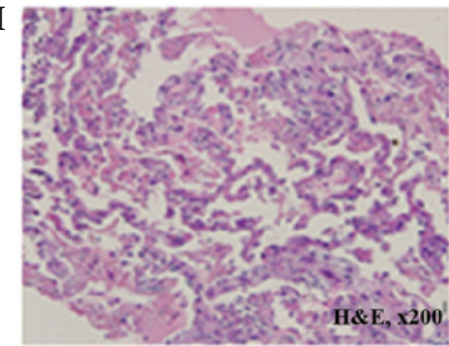

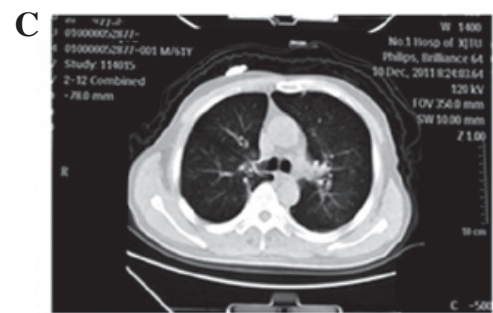

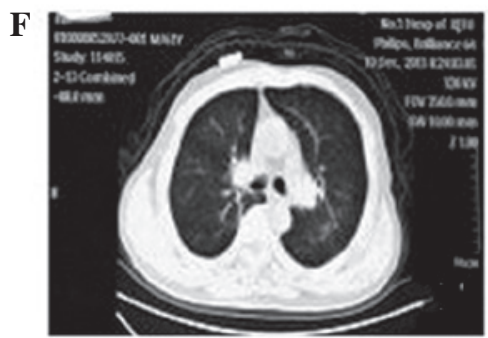

I

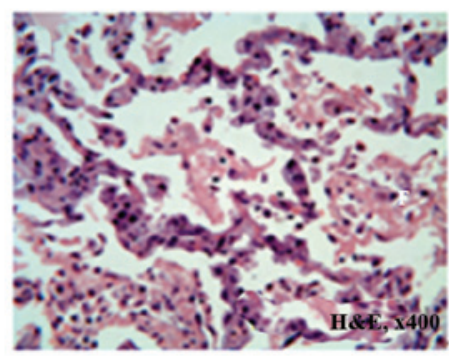

Figure 1. Primary lung tumor and interstitial pneumonia prior to and following treatment. (A) Prior to chemotherapy, multiple nodules were scattered in the double-lung field. (B) Following four cycles of chemotherapy, the therapeutic effect was progressive disease; (C) 30 days subsequent to the last administration of gefitinib, the therapeutic effect was partial response; (D) 60 days subsequent to the last administration of gefitinib, interstitial pneumonia occurred. (E) Following seven days of treatment, interstitial pneumonia was significantly relieved. (F) Results following 30 days of treatment; (G) results following one year of gefitinib treatment. ( $\mathrm{H}$ and I) Lung pathology following interstitial pneumonia, occurring 60 days subsequent to the last administration of gefitinib [hematoxylin and eosin staining; magnification, $\mathrm{x} 200(\mathrm{H})$ and $\mathrm{x} 400(\mathrm{I})$ ].

and lung hilar lymph nodes were not invaded (stage IB, pT2aNOM0). Following surgery, the patient did not receive any further treatment. In June 2011, the patient started to cough with obvious incentive; white phlegm was apparent, accompanied by intermittent bloody sputum. A chest computed tomography (CT) scan (Fig. 1A) showed multiple small nodules scattered throughout the patient's lungs, suggesting pulmonary metastasis. The patient developed a progressive disease following four cycles of chemotherapy with a pemetrexed-cisplatin regimen, starting in July 2011 (Fig. 1B). The EGFR gene mutation test showed a deletion in exon 19; however, there were no mutations in exons 18,20 or 21 . The K-RAS gene mutation test showed no mutations in exons 13 or 61. In November 2011, daily treatment with oral gefitinib (250 mg/day) was initiated and the patient achieved a partial response one month subsequently (Fig. 1C). However, 60 days subsequent to the initiation of the gefitinib treatment, the patient developed fever, an aggravated dry cough and dyspnea. On the visit to our clinic, the patient appeared acutely ill and presented with dyspnea, with a body temperature fluctuating between 37.6 and $38.8^{\circ} \mathrm{C}$. The patient was previously healthy and reported no history of chronic diseases, such as hypertension, heart disease and diabetes, and acute or chronic infection, such as hepatitis and tuberculosis. He had a smoking history of $>20$ years ( 40 cigarettes a day) and had quit smoking for 13 years, with a smoking index of 800 . Physical examination showed that the patient had a body temperature of $38.3^{\circ} \mathrm{C}$, a pulse rate of 124 beats/min, a respiratory rate of 41 breaths $/ \mathrm{min}$ and a blood pressure of 130/80 mmHg. Hypoxemia and an oxyhemoglobin saturation of $88 \%$ were detected using pulse oximetry and visible, large erythema and pimples, with scales and itching, were observed around the ankles and the dorsal surfaces of the elbow joint. The examination results are listed in Table I. A chest CT scan showed interstitial inflammation of the lungs, bilateral pleural effusion and an adverse swelling of the lungs (Fig. 1D). The patient was admitted with suspected gefitinib-induced interstitial pneumonitis.

Following admission, gefitinib administration was discontinued immediately. No evidence of infection or the presence of pathological microorganisms was found in either the sputum or blood culture (Table I), while chest CT revealed a diffuse ground-glass opacity over the whole right and left lungs. A lung biopsy revealed interstitial pneumonia, with a widening of the alveolar septa and alveolar epithelial hyperplasia. A small quantity of inflammatory exudate was observed in the alveolar cavity (Fig. 1H and I). The patient was treated with inhaled oxygen, electrocardiography, cefoperazone/sulbactam (3.0 g twice daily; to provide resistance to infection), doxofylline ( $0.2 \mathrm{~g}$ once daily; to relieve coughing), high-dose methylprednisolone $(1,000 \mathrm{mg}$ daily for three days), inhalation of expectorant and dexamethasone (5.0 mg twice daily), acetylcysteine (600 mg/Tid; to prevent pulmonary fibrosis) and an infusion of protein. Therapies for the protection of the liver and gastric mucosa, improvement of the immune system, prevention of fungal infections (fluorouracil mouthwash) and adjustment of the intestinal flora were 
Table I. Test results prior to and following treatment.

\begin{tabular}{|c|c|c|}
\hline Test & Prior to treatment & Following treatment \\
\hline \multicolumn{3}{|l|}{ Complete blood count } \\
\hline $\mathrm{WBC}\left(\mathrm{x} 10^{9} / \mathrm{l}\right)$ & 13.47 & 11.1 \\
\hline NEUT $(\%)$ & 76.00 & 50.74 \\
\hline \multicolumn{3}{|l|}{ Biochemical examination } \\
\hline $\operatorname{AST}(\mathrm{U} / \mathrm{l})$ & 131 & 63 \\
\hline $\operatorname{ALT}(\mathrm{U} / \mathrm{l})$ & 181 & 104 \\
\hline GGT (U/l) & 56 & 49 \\
\hline TBIL $(\mu \mathrm{mol} / 1)$ & 23.7 & 19.2 \\
\hline $\operatorname{DBIL}(\mu \mathrm{mol} / \mathrm{l})$ & 9.8 & 4.4 \\
\hline $\operatorname{ALB}(g / 1)$ & 33 & 23 \\
\hline \multicolumn{3}{|l|}{ Arterial blood } \\
\hline $\mathrm{PO}_{2}(\mathrm{mmHg})$ & 51.0 & 65.2 \\
\hline $\mathrm{PCO}_{2}(\mathrm{mmHg})$ & 27.0 & 32.1 \\
\hline $\mathrm{pH}$ & 7.440 & 7.415 \\
\hline ECG & sinus tachycardia & \\
\hline Blood culture & & $(-)$ \\
\hline GM Test & $(-)$ & \\
\hline Sputum culture & $(-)$ & $(-)$ \\
\hline PCT test $(\mathrm{ng} / \mathrm{ml})$ & $<0.5$ & \\
\hline Phlegm fungi smear & $(-)$ & $(-)$ \\
\hline \multicolumn{3}{|l|}{ Sputum smear } \\
\hline Gram positive coccus & ++1 & \\
\hline GNB & +1 & \\
\hline Phlegm fungi training & $(-)$ & $(-)$ \\
\hline
\end{tabular}

WBC, white blood cells; NEUT, neutrophils; AST, aspartate aminotransferase; ALT, alanine aminotransferase; GGT, gamma glutamyl transpeptidase; TBIL, total bilirubin; DBIL, direct bilirubin; ALB, albumin; $\mathrm{PO}_{2}$, partial pressue of oxygen; $\mathrm{PCO}_{2}$, partial pressure of carbon dioxide; ECG, electrocardiography; GM, galactomannan; PCT, procalcitonin; GNB, gram negative bacilli.

also administered to the patient. The patient's dyspnea and hypoxemia improved significantly in three days. The results of the laboratory tests taken 10 days subsequent to the treatments are presented in Table I. The follow-up high-resolution CT scan (Fig. 1E) showed the total resolution of the ground-glass lesions and the absorption of the right-side pleural fluid. Based on the patient's recovery, gefitinib treatment was reinitiated, under close observation. The gefitinib dosage was adjusted to $250 \mathrm{mg}$, taken once every two days. In addition, acetylcysteine was administered to prevent pulmonary fibrosis. No significant adverse response was detected in the patient. One month later, the follow-up chest CT (Fig. 1F) showed no interstitial pneumonia recurrence or tumor progression.

Literature review. Based on the case reports and literature reviews on interstitial pneumonia published in Western countries and China between 2003 and 2011, the clinical characteristics of gefitinib-induced interstitial pneumonia were preliminarily analyzed. The results are presented in Table II (7-29).

\section{Discussion}

Gefitinib is an oral selective inhibitor of the EGFR tyrosine kinase and may be effective in patients with advanced non-small-cell lung, ovarian, breast, head and neck or colon cancers. A significant survival benefit has been demonstrated for patients of Asian origin and non-smokers. At present, gefitinib is used as the second or third-line therapy for patients with locally advanced or metastatic NSCLC, following the failure of platinum and docetaxel-based chemotherapies, in a number of eastern Asian countries (30).

The most common adverse effects associated with the use of gefitinib are acneiform skin rashes, diarrhea and nausea, which are usually mild in severity and manageable (31). However, in addition to these effects, gefitinib-induced acute interstitial pneumonia is an infrequent but potentially lethal adverse effect. The precise mechanism of gefitinib-induced interstitial pneumonia remains unknown. Members of the EGF family have been demonstrated to be implicated in the repair of pulmonary damage (32). Therefore, inhibition of EGFR-mediated signaling by gefitinib may impair the repair of the bronchioloalveolar epithelium, thereby exacerbating lung injury, particularly in patients with pulmonary comorbidities (33). This may be one of the causes of gefitinib-induced ILD.

Although gefitinib-induced lung injury has a low incidence, the number of patients with gefitinib-induced lung injury is likely to increase. The incidence of ILD during gefitinib treatment varies among different ethnicities. The highest cumulative incidence of $4 \%$, following 12 weeks of gefitinib treatment, was described in a large Japanese cohort of $>1,800$ patients (34). In the rest of the world, including Taiwan, the incidence of ILD has been reported to be $1 \%$ (35).

The clinical manifestations of gefitinib-induced ILD consist of chest tightness, shortness of breath, progressive dyspnea, severe hypoxemia and respiratory failure. Gefitinib-induced ILD may be diagnosed according to previously published and generally accepted clinical criteria (36). In brief, a patient may be diagnosed with gefitinib-induced ILD if the following conditions are met: (i) There is a new onset of respiratory symptoms during gefitinib treatment; (ii) there are characteristic signs on the chest radiography or CT scan, such as non-specific areas with ground-glass attenuation and extensive bilateral ground-glass attenuation or airspace consolidations with traction bronchiectasis (31); (iii) exclusion of pulmonary infection and a progression of lung cancer, including lymphangitis and carcinomatosis; and (d) exclusion of radiation pneumonitis. To relieve the symptoms of gefitinib-induced ILD, there is a requirement for timely judgment and gefitinib withdrawal to be applied and for high-dose glucocorticoid (37), oxygen inhalation and anti-infective therapies to be administered.

The patient in this case report was described as being previously healthy, with a long history of smoking and a smoking index of 800 . The patient had ceased from smoking for 13 years, and was suffering from bronchioloalveolar carcinoma, which had metastasized to the lungs. Following the failure of the first-line treatments of pemetrexed plus cisplatin, EGFR and K-RAS gene results showed that the patient was suitable for the targeted therapy. One month subsequent to gefitinib treatment, the double-lung metastatic carcinoma was significantly relieved. An analysis of the domestic literature published over the past 
Table II. Clinical characteristics of gefitinib-induced interstitial pneumonia in case reports published from 2003 to 2011.

\begin{tabular}{|c|c|c|c|c|c|c|c|}
\hline Case no. & $\begin{array}{c}\text { Age } \\
\text { (years) }\end{array}$ & Gender & Pathology & $\begin{array}{l}\text { Smoking history } \\
\text { (years) }\end{array}$ & $\begin{array}{l}\text { Onset time (days } \\
\text { post-gefitinib } \\
\text { administration) }^{[\mathrm{ref}]}\end{array}$ & $\begin{array}{l}\text { Radiation } \\
\text { history }\end{array}$ & Prognosis \\
\hline 1 & 75 & Male & Adenocarcinoma & NA & $12^{[7]}$ & No & Relieved \\
\hline 2 & 60 & Male & Adenocarcinoma & NA & $40^{[8]}$ & 8 Gy & Deceased \\
\hline 3 & 55 & Male & Adenocarcinoma & NA & $210^{[9]}$ & No & Relieved \\
\hline 4 & 59 & Male & Adenocarcinoma & 20 & $23^{[10]}$ & No & Relieved \\
\hline 5 & 75 & Male & Adenocarcinoma & 14 & $2^{[11]}$ & No & Deceased \\
\hline 6 & 59 & Male & Adenocarcinoma & No & $60^{[12]}$ & No & Deceased \\
\hline 7 & 41 & Female & Adenocarcinoma & No & $20^{[13]}$ & No & Relieved \\
\hline 8 & 74 & Female & Adenocarcinoma & No & $5^{[14]}$ & No & Relieved \\
\hline 9 & 55 & Male & Adenocarcinoma & 35 & $42^{[15]}$ & No & Relieved \\
\hline 10 & 60 & Female & Adenocarcinoma & No & $34^{[16]}$ & No & Relieved \\
\hline 11 & 57 & Male & Adenocarcinoma & NA & $90^{[17]}$ & No & Relieved \\
\hline 12 & 74 & Female & Adenocarcinoma & No & $15^{[18]}$ & No & Relieved \\
\hline 13 & 77 & Female & Adenocarcinoma & NA & $20^{[19]}$ & No & Relieved \\
\hline 14 & 28 & Female & Adenocarcinoma & No & $25^{[20]}$ & No & Relieved \\
\hline 15 & 50 & Male & Adenocarcinoma & 30 & $38^{[21]}$ & $60 \mathrm{~Gy}$ & Relieved \\
\hline 16 & 65 & Male & Adenocarcinoma & 40 & $2^{[22]}$ & No & Deceased \\
\hline 17 & 73 & Male & Squamous carcinoma & Long & $60^{[23]}$ & No & Relieved \\
\hline 18 & 81 & Male & Adenocarcinoma & Long & $13^{[24] \mathrm{a}}$ & No & Deceased \\
\hline 19 & 66 & Female & Adenocarcinoma & No & $55^{[25]}$ & No & Relieved \\
\hline 20 & 51 & Female & Adenocarcinoma & No & $56^{[26]}$ & No & Relieved \\
\hline 21 & 70 & Male & Adenocarcinoma & No & $43^{[27]}$ & $44 \mathrm{~Gy}$ & Relieved \\
\hline 22 & 58 & Female & Adenocarcinoma & No & $38^{[28]}$ & No & Relieved \\
\hline 23 & 79 & Male & Adenocarcinoma & NA & $25^{[29]}$ & No & Deceased \\
\hline
\end{tabular}

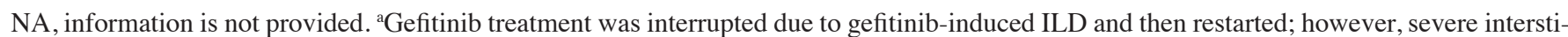
tial pneumonia occurred 13 days subsequent to the restart of the treatment. Cases 1-14, data from case reports published in Western countries (references present in Pubmed); 15-23, data from case reports published in China (references not present in Pubmed).

few years has shown the importance of gender, pathological type and smoking history among the factors affecting response rate and prognosis. Female patients with adenocarcinoma (particularly with alveolar cell carcinoma) and no smoking history have a good prognosis and experience an improved quality of life following gefitinib treatment. However, the present case showed that satisfactory efficacy may also be achieved in male patients with adenocarcinoma and a long-term smoking history, providing the results of the genetic testing are in accordance with the indications for gefitinib. This case report indicated that the EGFR mutation status may be the ultimate decisive factor affecting the response rate and prognosis for gefitinib treatment.

Adenocarcinoma and individual squamous cell carcinoma have been revealed to be the most common types of gefitinib-induced interstitial pneumonia. A total of 14 case reports of gefitinib-induced interstitial pneumonia that have been published in Western countries were reviewed. These cases each had individual characteristics; however, the majority concerned male smokers with adenocarcinoma. In addition, nine cases of gefitinib-induced interstitial pneumonia were analyzed from the studies published between 2004 and 2011 in China. The patients in these cases shared certain features, such as predominantly being female, with no history of smoking and with adenocarcinoma and a radiation history. These features are correlated with a low risk of ILD occurrence, a high response rate and a long survival. The results of this analysis are consistent with the results of analyses of gefitinib-induced interstitial pneumonia that have been produced in Western countries (38). The time of ILD-onset has been shown to range between 2 and 60 days following gefitinib treatment (Table II).

Following treatment, the patient in the present case recovered and continued to take gefitinib under careful medical supervision. The patient was still alive, one year subsequent to the restart of the EGFR tyrosine kinase inhibitor therapy. The outcome of this case showed that the occurrence of controllable ILD is not an absolute index for the discontinuation of gefitinib administration. The benefits to the patient and the risks associated with the use of the drug require comprehensive consideration prior to the decision regarding the continuation or discontinuation of gefitinib therapy being made.

Case 18 in Table II continued gefitinib treatment following an interruption; however, severe interstitial pneumonia occurred 13 days subsequent to the restart of the treatment, and the patient died. It was presumed that the gefitinib-induced ILD in this patient was immune-related and that, following the first dose of gefitinib, antibodies were induced in the patient. 
When the patient took the same drug again, subsequent to the temporary discontinuation, an antigen-antibody reaction took place, resulting in the formation of immune complexes and the onset of ILD. Similar conditions were not observed in the patient in the present case, which indicated that individual differences exist in the response to gefitinib.

In conclusion, when gefitinib is used to treat advanced NSCLC, it confers a high risk of ILD in patients with progressionfree survival and a significant clinical benefit in non-smokers, females, patients with adenocarcinoma and patients with no history of thoracic radiotherapy. Gefitinib therapy is an important treatment option for patients with advanced NSCLC; however, physicians should carefully decide on the indications for the use of gefitinib and other cytotoxic agents, particularly for patients with lung comorbidities. In addition, it is necessary for careful attention to be paid to the clinical respiratory symptoms of the patients and the radiographic results, particularly during the first 1-2 months following the initiation of gefitinib treatment.

\section{References}

1. Von Pawel J: Gefitinib (Iressa, ZD 1839): a novel targeted approach for the treatment of solid tumors. Bull Cancer 91: E70-E76, 2004.

2. Cerosimo RJ: Gefitinib: an adverse effects profile. Expert Opin Drug Saf 5: 469-479, 2006.

3. Fukuoka M, Yano S, Giaccone G, et al: Multi-institutional randomized phase II trial of gefitinib for previously treated patients with advanced non-small-cell lung cancer. J Clin Oncol 21: 2237-2246, 2003.

4. Giaccone G, Herbst RS, Manegold C, et al: Gefitinib in combination with gemcitabine and cisplatin in advanced non-small-cell lung cancer: a phase III trial - INTACT 1. J Clin Oncol 22 777-784, 2004

5. Herbst RS, Giaccone G, Schiller JH, et al: Gefitinib in combination with paclitaxel and carboplatin in advanced non-small-cell lung cancer: a phase III trial - INTACT 2. J Clin Oncol 22: 785-794, 2004

6. Ohyanagi F, Ando Y, Nagashima F, et al: Acute gefitinib-induced pneumonitis. Int J Clin Oncol 9: 406-409, 2004.

7. Teramoto S, Yamamoto $\mathrm{H}$ and Ouchi Y: Clinical efficacy and toxicity of gefitinib in patients with lung cancer. Lancet 361: 1992-1993, 2003.

8. Sumpter K, Harper-Wynne C, O'Brien M and Congleton J: Severe acute interstitial pneumonia and gefitinib. Lung Cancer 43 : 367-368, 2004.

9. Umemura S, Kishino D, Tabata M, et al: Systemic tumor embolism mimicking gefitinib ('IRESSA')-induced interstitial lung disease in a patient with lung cancer. Intern Med 44: 979-982, 2005.

10. Suzuki M, Asahina H,Konishi J, et al: Recurrent gefitinib-induced interstitial lung disease. Intern Med 47: 533-536, 2008.

11. Aoe K, Hiraki A, Murakami T, et al: Sudden onset of interstitial lung disease induced by gefitinib in a lung cancer patient with multiple drug allergy. Anticancer Res 25: 415-418, 2005.

12. Nagaria NC, Cogswell J, Choe JK and Kasimis B: Side effects and good effects from new chemotherapeutic agents. Case 1. Gefitinib-induced interstitial fibrosis. J Clin Oncol 23: 2423-2424, 2005 .

13. Tian Q and Chen LA: Erlotinib achieved partial response in a non-small cell lung cancer patient with gefitinib-induced interstitial lung disease. Case Rep Oncol 4: 464-466, 2011.

14. Kuo LC, Lin PC, Wang KF, et al: Successful treatment of gefitinib-induced acute interstitial pneumonitis with high-dose corticosteroid: a case report and literature review. Med Oncol 28: 79-82, 2011.

15. Seto T, Seki N, Uematsu K, et al: Gefitinib-induced lung injury successfully treated with high-dose corticosteroids. Respirology 11: 113-116, 2006.

16. Ohyanagi F, Ando Y, Nagashima F, Narabayashi M and Sasaki Y: Acute gefitinib-induced pneumonitis. Int J Clin Oncol 9: 406-409, 2004.
17. Kitajima H, Takahashi H, Harada K, et al: Gefitinib-induced interstitial lung disease showing improvement after cessation: disassociation of serum markers. Respirology 11: 217-220, 2006.

18. Sakoda Y, Kitasato Y, Kawano Y, et al: A case of alveolar hemorrhage caused by gefitinib. Nihon Kokyuki Gakkai Zasshi 49: 506-510, 2011 (In Japanese).

19. Goto Y, Hojo M, Takeda Y, et al: Gefitinib-induced interstitial lung disease - addition of intravenous cyclophosphamide to corticosteroids is a valuable treatment option: A case report. Med Oncol 27: 753-755, 2010.

20. Fukui T, Otani S, Hataishi R, et al: Successful rechallenge with erlotinib in a patient with EGFR-mutant lung adenocarcinoma who developed gefitinib-related interstitial lung disease. Cancer Chemother Pharmacol 65: 803-806, 2010.

21. Su Y, Peng L and Wu YM: A case of gefitinib maintenance treatment of non-small cell lung cancer caused by interstitial pneumonia. Chongqing Medical Journal 40: 310, 2011 (In Chinese)

22. Peng W, Gao J and Miao LY: Death from pulmonary interstitial fibrosis and liver damage after taking gefitinib. Adverse Drug Reactions Journal 11: 429-430, 2009 (In Chinese).

23. Feng ZZ, Cao Y, Sun LM and Sun P: A cases of Gefitinib treatment of non-small cell lung cancer with finger bone metastasis caused interstitial pneumonia. Journal of Dalian Medical University 30: 588-589, 2008 (In Chinese).

24. Cui H, Huang Q and Chen Y: A case report of Gefitinib-induced interstitial pneumonia. J Clin Oncol 12: 235-237, 2007.

25. Liang $\mathrm{H}$ and Ynag $\mathrm{H}$ : A case of Gefitinib-induced interstitial pneumonia. Practical Journal of Medicine 1: 162, 2009.

26. Huang JH, Zhang Y and Zheng J: Interstitial pneumonia caused by gefitinib in one patient. Chinese Journal of New Drugs and Clinical Remedies 26: 878-879, 2007 (In Chinese).

27. Wang WL and Sun Y: One case of interstitial pneumonia caused by oral gefitinib and radiotherapy. Pharmaceutical and Clinical Research 16: 506-507, 2008 (In Chinese).

28. Zhou Y, Dai J, Zhu YZ and Chen FL: Gefitinib-induced interstitial pneumonia CT image analysis. China Modern Doctor 12: 61-62, 2010 (In Chinese).

29. Cao SF, Deng QN and Zhang DM: One case of epidermal growth factor receptor inhibitors-IRESA induced interstitial pneumonia. Journal of Guangdong College of Pharmacy 20: 304-305, 2004 (In Chinese).

30. Ciardiello $\mathrm{F}$ and Tortora G: EGFR antagonists in cancer treatment. N Engl J Med 358: 1160-1174, 2008.

31. Hotta K, Kiura K, Takigawa N, et al: Comparison of the incidence and pattern of interstitial lung disease during erlotinib and gefitinib treatment in Japanese patients with non-small cell lung cancer: the Okayama Lung Cancer Study Group experience. J Thorac Oncol 5: 179-184, 2010.

32. Min JH, Lee HY, Lim H, et al: Drug-induced interstitial lung disease in tyrosine kinase inhibitor therapy for non-small cell lung cancer: a review on current insight. Cancer Chemother Pharmacol 68: 1099-1109, 2011.

33. Danson S, Blackhall F, Hulse P and Ranson M: Interstitial lung disease in lung cancer: separating disease progression from treatment effects. Drug Saf 28: 103-113, 2005.

34. Kudoh S, Kato H, Nishiwaki Y, et al: Interstitial lung disease in Japanese patients with lung cancer: a cohort and nested case-control study. Am J Respir Crit Care Med 177: 1348-1357, 2008.

35. Armour A: Gefitinib in advanced non-small cell lung cancer: Clinical experience in patients of Asian origin. Asia Pac J Clin Oncol 3: 66-78, 2007.

36. American Thoracic Society; European Respiratory Society: American Thoracic Society/European Respiratory Society International Multidisciplinary Consensus Classification of the Idiopathic Interstitial Pneumonias. This joint statement of the American Thoracic Society (ATS), and the European Respiratory Society (ERS) was adopted by the ATS board of directors, June 2001 and by the ERS Executive Committee, June 2001. Am J Respir Crit Care Med 15: 277-304, 2002.

37. Zhang Y, Yang H, Zhao M and He J: Successful treatment of gefitinib-induced acute interstitial pneumonitis with corticosteroid and non-invasive BIPAP-ventilation. J Thorac Dis 4: 316-319, 2012.

38. Shih YN, Chiu CH, Tsai CM and Perng RP: Interstitial pneumonia during gefitinib treatment of non-small-cell lung cancer. J Chin Med Assoc 68: 183-186, 2005. 